\title{
The method of finite-product extraction and an application to Wiener-Hopf theory
}

\author{
ANTHONY D. RAWLINS \\ Department of Mathematical Science, Brunel University, \\ Kingston Lane, Uxbridge, UB8 3PH, UK.
}

\begin{abstract}
In this work we describe a simple method for f nding approximate representations for special functions which are entire transcendental functions that can be represented by inf nite products. This method replaces the inf nite product by a f nite polynomial and Gamma functions. This approximate representation is shown in the case of Bessel functions to be very accurate over a large range of parameter values. These approximate expressions can be useful for $\mathrm{f}$ nding the roots of a transcendental equation and the WienerHopf factorization of functions involving such Bessel functions. The method is shown to be potentially useful for other transcendental and Wiener-Hopf problems, which involve other entire functions that have inf nite product representations.
\end{abstract}

Keywords: Transcendental equations, [roots, Lzeros, $\mathbb{B}$ Bessel Functions,Lpolynomial approximations, WienerHopf factorization.

\section{Introduction}

The inspiration for this work arose from reading the article (Chapman (2010)) in which the authors found that by replacing trigonometric functions by a f nite product polynomial and a Gamma function expression they were able to get very good approximations for the roots of eigenvalue equations. The replacement was exact and followed from the well known identities between the Gamma function and trigonometric functions. We propose here to replace any transcendental function that is an entire function and that can be represented by an inf nite product by an approximate expression that involves a polynomial and a function of Gamma functions. Initially to describe this extension of the method of f nite-product extraction we apply the method to derive an approximation for the Bessel function $J_{v}(z)$. We use this approximation to f nd the roots of a transcendental equation that involves Bessel functions and check the numerical accuracy of the approximation. In many physical applications one often needs to consider the roots of Bessel function equations of the form

$$
\beta J_{v}(z)+z^{\alpha} J_{\mu}^{\prime}(z)=0
$$

where $J_{v}(z)\left(J_{\mu}(z)\right)$ is the Bessel function of the frst kind of order $v(\mu)$ and $\beta$, and $\alpha$ are parameters possibly complex. This is a fairly general equation that arises in numerous applications involving cylindrical boundary value problems. For example special cases of this equation arise as an eigenvalue equations in acoustically lined ducts:

$$
\beta J_{v}(z)+z J_{v}^{\prime}(z)=0,
$$

see Rawlins (2007) and references therein. An equation of the form :

$$
\beta J_{v}(z)+J_{v}^{\prime}(z)=0 .
$$


arises for the vibration of a circular membrane with elastic boundary support Chen \& Zhou (1993) and for tsunami wave generation, see references in Synolakis (1988) We also show how we can carry out an approximate Wiener-Hopf ( Lawrie \& Abrahams (2007)) factorization for a Bessel function expression. We then outline a generalization of the method of $\mathrm{f}$ nite-Product extraction to a general class of entire function. These results will have potential applications for other transcendental equations and WienerHopf factorization problems in the future.

\section{An approximation for Bessel functions}

We use the Bessel function representation given in Abramowitz (1964),(page 370, 9.5.10) and Watson (1944), (page 498)

$$
J_{v}(z)=\frac{\left(\frac{z}{2}\right)^{v}}{\Gamma(v+1)} \prod_{n=1}^{\infty}\left[1-\left(\frac{z}{j_{v, n}}\right)^{2}\right] .
$$

where $j_{v, n}$ is the n-th nonnegative zero of the Bessel function of order $v$. We begin by splitting the inf nite product at the value $n=m-1$ giving

$$
J_{v}(z)=\frac{\left(\frac{z}{2}\right)^{v}}{\Gamma(v+1)} \prod_{n=1}^{m-1}\left[1-\left(\frac{z}{j_{v, n}}\right)^{2}\right] \prod_{n=m}^{\infty}\left[1-\left(\frac{z}{j_{v, n}}\right)^{2}\right] .
$$

Provided we make $\mathrm{m}$ large enough the roots in second inf nite product can be approximated by their asymptotic form:

$$
j_{v, n}=\left(n+\frac{v}{2}-\frac{1}{4}\right) \pi+O\left(\frac{1}{N}\right)
$$

as $N=\left(n+\frac{v}{2}-\frac{1}{4}\right) \pi \rightarrow \infty$, see Abramowitz (1964),(page 371, 9.5.12). This being so we can approximate the Bessel function by the expression:

$$
J_{v}(z) \simeq \frac{\left(\frac{z}{2}\right)^{v}}{\Gamma(v+1)} \prod_{n=1}^{m-1}\left[1-\left(\frac{z}{j_{v, n}}\right)^{2}\right] \prod_{n=m}^{\infty}\left[1-\frac{z^{2}}{\left(\left(n+\frac{v}{2}-\frac{1}{4}\right) \pi\right)^{2}}\right] .
$$

which we can write as

$$
J_{v}(z) \simeq \frac{\left(\frac{z}{2}\right)^{v}}{\Gamma(v+1)} \prod_{n=1}^{m-1}\left[1-\left(\frac{z}{j_{v, n}}\right)^{2}\right] \prod_{k=1}^{\infty}\left[1-\frac{(z / \pi)^{2}}{\left(k+m+\frac{v}{2}-\frac{5}{4}\right)^{2}}\right] .
$$

By using an identity, that can be easily derived from the result Erdelyi (1953),(p5, 1.3(4)):

$$
\prod_{k=1}^{\infty}\left[1-\frac{\zeta^{2}}{(a k+b)^{2}}\right]=\frac{\Gamma^{2}(1+b / a)}{\Gamma\left(1+\frac{b+\zeta}{a}\right) \Gamma\left(1+\frac{b-\zeta}{a}\right)} .
$$

we can write $(2.6)$ as $J_{v}(z) \simeq \widetilde{J}_{v}(z)$ where

$$
\widetilde{J}_{v}(z)=\frac{\left(\frac{z}{2}\right)^{v}}{\Gamma(v+1)} \frac{\Gamma^{2}\left(m+\frac{v}{2}-\frac{1}{4}\right)}{\Gamma\left(m+\frac{v}{2}-\frac{1}{4}+\frac{z}{\pi}\right) \Gamma\left(m+\frac{v}{2}-\frac{1}{4}-\frac{z}{\pi}\right)} \prod_{n=1}^{m-1}\left[1-\left(\frac{z}{j_{v, n}}\right)^{2}\right],
$$

which can be regarded as a good approximation for $\left(m+\frac{v}{2}-\frac{1}{4}\right) \gg 1$. In an exactly analogous manner from the results in Abramowitz (1964)(page 370, 9.5.11; and page 371, 9.15.13) it can be shown that for 
$v>0$, and $\left(m+\frac{v}{2}-\frac{3}{4}\right) \gg 1$, then the derivative of the Bessel function of order $v$ can be approximated by the expression $J_{v}^{\prime}(z) \simeq \widetilde{J}^{\prime}{ }_{v}(z)$ where:

$$
\widetilde{J}_{v}^{\prime}(z)=\frac{\left(\frac{z}{2}\right)^{v-1}}{2 \Gamma(v)} \frac{\Gamma^{2}\left(m+\frac{v}{2}-\frac{3}{4}\right)}{\Gamma\left(m+\frac{v}{2}-\frac{3}{4}+\frac{z}{\pi}\right) \Gamma\left(m+\frac{v}{2}-\frac{3}{4}-\frac{z}{\pi}\right)} \prod_{n=1}^{m-1}\left[1-\left(\frac{z}{j_{v, n}^{\prime}}\right)^{2}\right] .
$$

As a demonstration of the accuracy of this approximation we display some typical graphs below. In Figures 1-7 the top plot is of $J_{v}(z)$ and $\widetilde{J}_{v}(z)$ superimposed on each other over the range $0<z<10$ for $v=0$, and $0<z<100$ for $v=8$. In the lower plots the difference $J_{v}(z)-\widetilde{J}_{v}(z)$ is plotted over the range $0<z<100$. The plots in Figure 1-3 are for $v=0$, and the plots in Figure 4-7 are for the larger value of $v=8$.

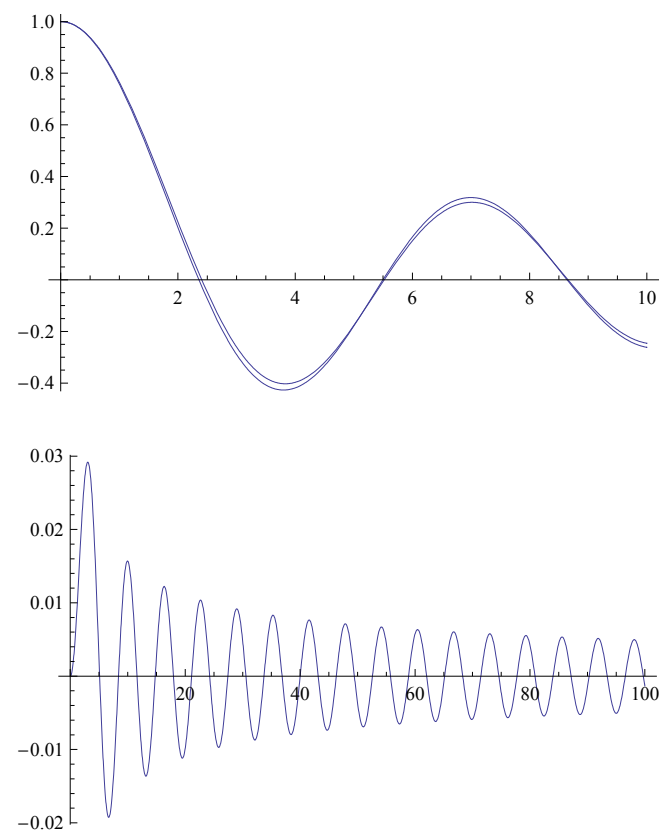

FIG. 1. The error between approximate and exact expressions $J_{0}(z)-\widetilde{J}_{0}(z)$, for $v=0, m=1$. 

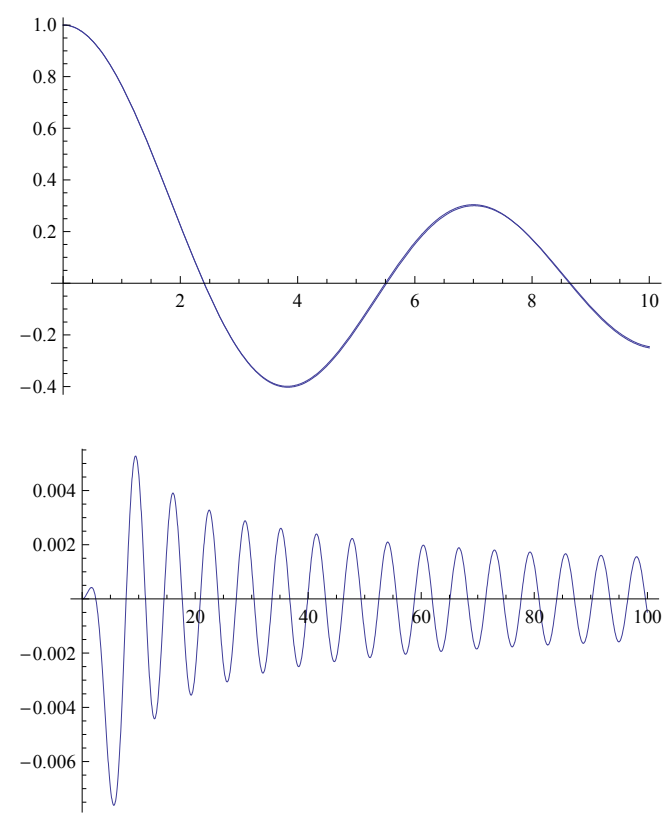

FIG. 2. The error between approximate and exact expressions $J_{0}(z)-\widetilde{J}_{0}(z)$, for $v=0, m=3$.
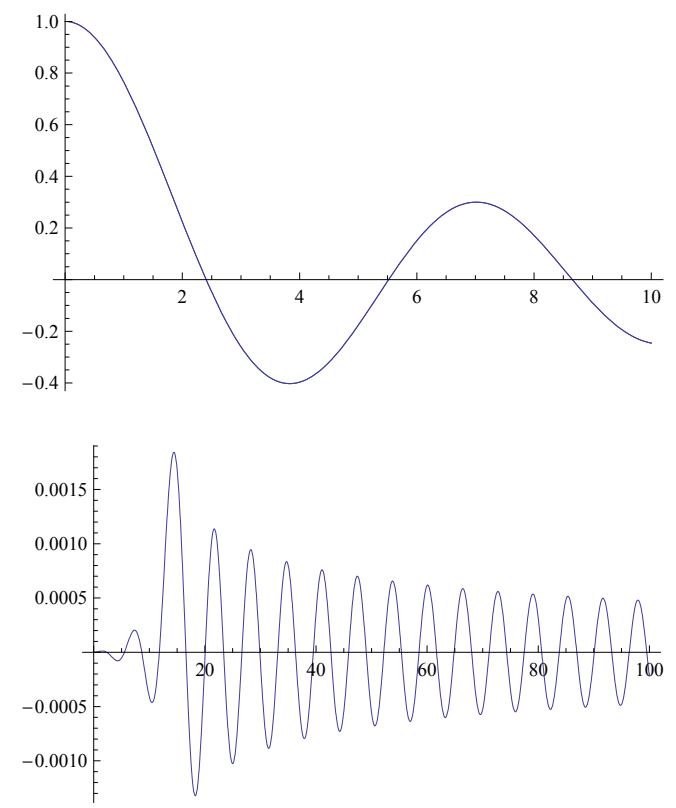

FIG. 3. The error between approximate and exact expressions $J_{0}(z)-\widetilde{J}_{0}(z)$, for $v=0, m=5$. 

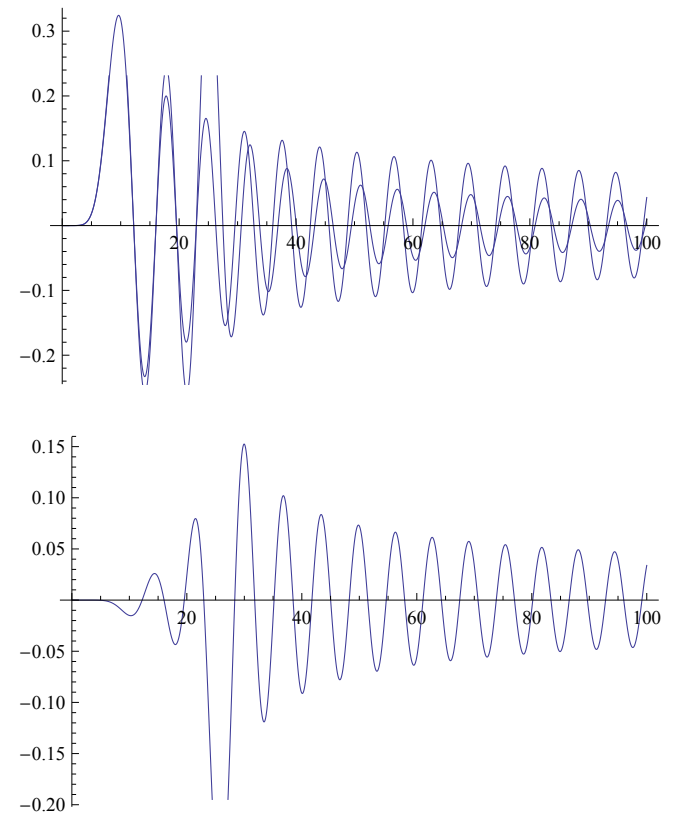

FIG. 4. The error between approximate and exact expressions $J_{8}(z)-\widetilde{J}_{8}(z)$, for $v=8, m=5$.
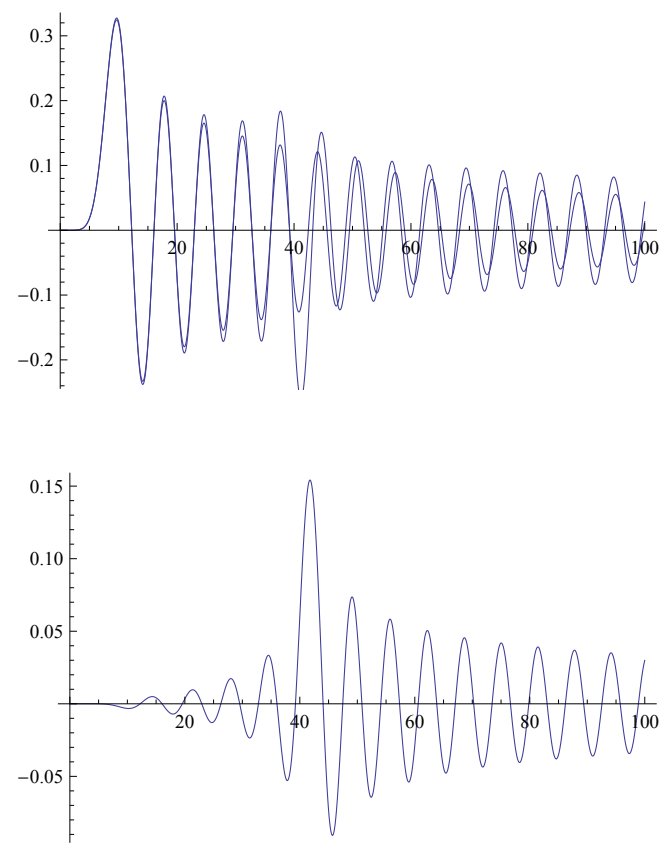

FIG. 5. The error between approximate and exact expressions $J_{8}(z)-\widetilde{J}_{8}(z)$, for $v=8, m=10$. 

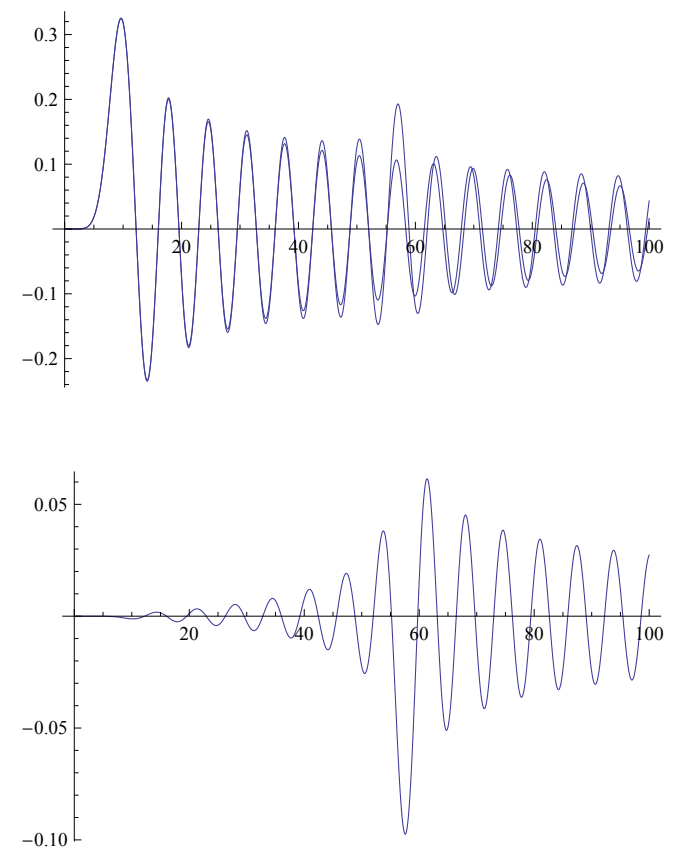

FIG. 6. The error between approximate and exact expressions $J_{8}(z)-\widetilde{J}_{8}(z)$, for $v=8, m=15$. 

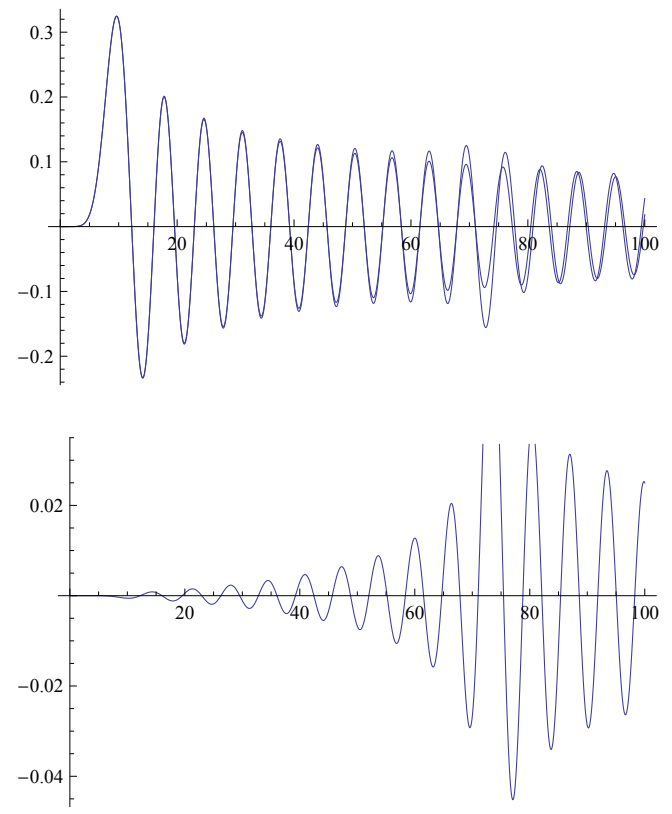

FIG. 7. The error between approximate and exact expressions $J_{8}(z)-\widetilde{J_{8}}(z)$, for $v=8, m=20$. 
From these graphs it can be seen that as we increase $m$, i.e increasing the degree of the polynomial, we improve the accuracy near $z=0$. The large $z$ behaviour is well approximated by the Gamma functions for large enough values of $m$. The approximation can be made good within prescribed bounds over the entire range of values of $z$ by choosing a suitably large value of $m$. In practice this value of $m$ is surprisingly small for small $v$ ! The agreement is not so good for all $z$ for larger values of $v$, and this is could be due to the fact that Bessel function approximations for large order are only good for $z>v^{2}$.

\section{Some numerical examples of the Bessel function approximation}

These type of results were obtained for a wide range of complex values of the parameter $\beta$, for $\alpha=0,1$ in equation (1). We f rst apply the approximations to the roots $z_{n}, n=1,2,3 \ldots$, of the transcendental equation:

$$
J_{0}(z)+2 i J_{1}(z)=0
$$

and its approximate version:

$$
\widetilde{J}_{0}(z)+2 i \widetilde{J}_{1}(z)=0,
$$

whose corresponding roots are denoted by $\widetilde{z}_{n}, n=1,2,3 \ldots$. The same Newton's method with Mathematica is used to calculate the roots of (3.1) and (3.2). The $\mathrm{f}$ rst three roots of the equation (3.1) with the ordered magnitude $\left(0<\left|z_{1}\right|<\left|z_{2}\right|<\left|z_{3}\right|, \Re\left(z_{n}\right)>0\right)$ are given by $z_{1}=2.46633-0.52998 i, z_{2}=$ $5.54951-0.545114 i, z_{3}=8.67279-0.547564 i$. For the approximate equation (3.2) we obtain the corresponding approximate roots and error estimates:

\begin{tabular}{||l|r|r|r||}
\hline$m$ & 1 & 3 & 5 \\
\hline$\widetilde{z}_{1}$ & $2.39773-0.536855 i$ & $2.46618-0.530725 i$ & $2.46631-0.530116 i$ \\
\hline$z_{1}-\widetilde{z}_{1}$ & $0.06860+0.00687 i$ & $0.00015+0.00074 i$ & $0.00002+0.00013 i$ \\
\hline
\end{tabular}

\begin{tabular}{||l|r|r|r||}
\hline$m$ & 1 & 3 & 5 \\
\hline$\widetilde{z}_{1}$ & $5.50061-0.475500 i$ & $5.54866-0.550255 i$ & $5.54944-0.545877 i$ \\
\hline$z_{2}-\widetilde{z}_{2}$ & $0.03890-0.069600 i$ & $0.00085+0.005141 i$ & $0.00007+0.000763 i$ \\
\hline
\end{tabular}

\begin{tabular}{||l|r|r|r||}
\hline$m$ & 1 & 3 & 5 \\
\hline$\widetilde{z}_{1}$ & $8.64009-0.466037 i$ & $8.64998-0.563053 i$ & $8.67259-0.549758 i$ \\
\hline$z_{3}-\widetilde{z}_{3}$ & $0.03270+0.081428 i$ & $0.02281+0.015489 i$ & $0.00020+0.002194 i$ \\
\hline
\end{tabular}

We now apply the approximations to the roots $z_{n}, n=1,2,3 \ldots$, of the transcendental equation:

$$
z J_{0}^{\prime}(z)+2 i J_{0}(z)=0
$$

and its approximate version:

$$
z \widetilde{J}_{0}^{\prime}(z)+2 i \widetilde{J}_{0}(z)=0
$$

whose corresponding roots will be denoted by $\widetilde{z}_{n}, n=1,2,3 \ldots$. For the $\mathrm{f}$ rst root of this equation, given by $z_{1}=3.88737-0.566309 i$, we obtain the approximations 


\begin{tabular}{||l|r|r|r||}
\hline$m$ & 1 & 3 & 5 \\
\hline$\widetilde{z}_{1}$ & $1.67973-0.931529 i$ & $3.88757-0.564116 i$ & $3.88739-0.565936 i$ \\
\hline$z_{1}-\widetilde{z}_{1}$ & $2.20764+0.375220 i$ & $-0.00020-0.002193 i$ & $0.00002-0.000373 i$ \\
\hline
\end{tabular}

Results of similar accuracy have been obtained for other parameter values of $\beta$ in the equations (1.2) and (1.3), for example the equation studied in the works of Synolakis (1988) and Rawlins (1989).

\section{An example of an approximate Wiener-Hopf factorization for Bessel functions}

The Bessel function approximation also offers a simple way to give analytic representations for WienerHopf factorizations of Bessel functions. For example suppose we require that

$$
F(z)=F_{+}(z) F_{-}(z),
$$

where $F_{ \pm}(z)$ is analytic and non-zero in the respective half planes $\mathfrak{I} z \gtrless 0$.Thus for example if we let $F(z)=J_{v}(\kappa)$, where $\kappa=\sqrt{k^{2}-z^{2}}$, with $\Re \kappa>0$ in the cut $z$-plane, then from the result ( 2.7) by inspection we have the approximate factorization

$$
\widetilde{F}_{ \pm}(z)=\frac{(k \pm z)^{v / 2}}{\sqrt{2^{v} \Gamma(v+1)}} \prod_{n=1}^{m-1}\left[1 \pm \frac{\kappa}{j_{v, n}}\right] \frac{\Gamma\left(m+\frac{v}{2}-\frac{1}{4}\right)}{\Gamma\left(m+\frac{v}{2}-\frac{1}{4} \pm \frac{\kappa}{\pi}\right)}
$$

\section{Generalization for other entire transcendental functions with an Euler-Weierstrass product representation}

If $F(z)$ is an integral (entire) function of $z$ with simple zeros it can be represented in the form

$$
F(z)=F(0) e^{z \frac{F^{\prime}(0)}{F(0)}} \prod_{k=1}^{\infty}\left(1-\frac{z}{z_{k}}\right) e^{\frac{z}{z_{k}}}
$$

see Titchmarsh (1958), (p113). We write this in a more convenient alternative form where the zeros $z_{p}\left(-z_{n}\right)$ are such that $0 \leqslant \arg \left(z_{p}\right)<\pi\left(-\pi \leqslant \arg \left(-z_{n}\right)<0\right)$ and are ordered in an appropriate manner, with the asymptotic behaviour $z_{n} \rightarrow a n+b$ as $n \rightarrow \infty\left(z_{p} \rightarrow \alpha p+\beta\right.$ as $\left.p \rightarrow \infty\right)$. Then we can write the last product representation as

$$
F(z)=F(0) e^{z \frac{F^{\prime}(0)}{F(0)}} \prod_{p=1}^{\infty}\left(1-\frac{z}{z_{p}}\right) e^{\frac{z}{z_{p}}} \prod_{n=1}^{\infty}\left(1+\frac{z}{z_{n}}\right) e^{-\frac{z}{z_{n}}}
$$

We now extract the f nite products and use the asymptotic form for the roots giving the approximation for $F(z)$

$$
\begin{aligned}
\widetilde{F}(z) & =F(0) e^{\frac{F^{\prime}(0)}{F(0)}} \prod_{p=1}^{P-1}\left(1-\frac{z}{z_{p}}\right) e^{\frac{z}{z p}} \prod_{p=P}^{\infty}\left(1-\frac{z}{\alpha p+\beta}\right) e^{\frac{z}{\alpha p+\beta}} \\
& \times \prod_{n=1}^{N-1}\left(1+\frac{z}{z_{n}}\right) e^{-\frac{z}{z_{n}}} \prod_{n=N}^{\infty}\left(1+\frac{z}{a n+b}\right) e^{-\frac{z}{a n+b}}
\end{aligned}
$$


or

$$
\begin{aligned}
\widetilde{F}(z) & =F(0) e^{z \frac{F^{\prime}(0)}{F(0)}} \prod_{p=1}^{P-1}\left(1-\frac{z}{z_{p}}\right) e^{\frac{z}{z p}} \prod_{s=0}^{\infty}\left(1-\frac{z}{\alpha s+(\alpha P+\beta)}\right) e^{\frac{z}{\alpha s+(\alpha P+\beta)}} \\
& \times \prod_{n=1}^{N-1}\left(1+\frac{z}{z_{n}}\right) e^{-\frac{z}{z_{n}}} \prod_{s=0}^{\infty}\left(1+\frac{z}{a s+(a N+b)}\right) e^{-\frac{z}{a s+(a N+b)}}
\end{aligned}
$$

We now need to eliminate the inf nite products. This is achieved by using properties of the related Psi function and the Gamma function. Thus we note that since

$$
\psi(x)=\frac{d}{d x} \ln \Gamma(x)=\frac{\Gamma^{\prime}(x)}{\Gamma(x)}=-C-\frac{1}{x}+\sum_{n=1}^{\infty}\left(\frac{1}{n}-\frac{1}{x+n}\right),
$$

where $C$ is Euler's constant, then

$$
e^{y \psi(x)}=e^{-y C-\frac{y}{x}} \prod_{n=1}^{\infty} e^{\frac{y}{n}-\frac{y}{x+n}}
$$

and since

$$
\frac{\Gamma(x)}{\Gamma(x+y)}=e^{y C}\left(1+\frac{y}{x}\right) \prod_{n=1}^{\infty}\left(1+\frac{y}{x+n}\right) e^{-\frac{y}{n}},
$$

then eliminating the term involving the Euler constant $\mathrm{C}$ from the last two results we get Mellin's result, Erdelyi (1953) (p6, 1.3(5))

$$
\prod_{n=0}^{\infty}\left(1+\frac{y}{x+n}\right) e^{-\frac{y}{x+n}}=\frac{\Gamma(x)}{\Gamma(x+y)} e^{y \psi(x)} .
$$

Now let $y=z / a, x=b / a$ and we then get the identity

$$
\prod_{n=0}^{\infty}\left(1+\frac{z}{a n+b}\right) e^{-\frac{z}{a n+b}}=\frac{\Gamma\left(\frac{b}{a}\right)}{\Gamma\left(\frac{z+b}{a}\right)} e^{\frac{z}{a} \psi\left(\frac{b}{a}\right)} .
$$

We now use this last result to eliminate the inf nite products that appear in (5.2) giving the f nite product representation for $F(z)$ as

$$
\begin{aligned}
\widetilde{F}(z) & =F(0) \frac{\Gamma\left(\frac{b}{a}\right) \Gamma\left(\frac{\beta}{\alpha}\right)}{\Gamma\left(\frac{b+z}{a}\right) \Gamma\left(\frac{\beta-z}{\alpha}\right)} e^{z\left[\frac{F^{\prime}(0)}{F(0)}+\psi(N+b / a) / a-\psi(P+\beta / \alpha) / \alpha\right]} \\
& \times \prod_{p=1}^{P-1}\left(1-\frac{z}{z_{p}}\right) e^{\frac{z}{z p}} \prod_{n=1}^{N-1}\left(1+\frac{z}{z_{n}}\right) e^{-\frac{z}{z_{n}}} .
\end{aligned}
$$

This result simplif es considerably when $F(z)$ is an even function of $z$, in which case it reduces to

$$
\widetilde{F}(z)=F(0) \frac{\left(\Gamma\left(\frac{b}{a}\right)\right)^{2}}{\Gamma\left(\frac{b+z}{a}\right) \Gamma\left(\frac{b-z}{a}\right)} \prod_{n=1}^{N-1}\left(1-\left(\frac{z}{z_{n}}\right)^{2}\right) .
$$

The optimum choice of $P$ and $N$ in the above expressions will depend on the physical applications. For example in wave propagation in ducts $P$ and $N$ will be closely related to the number of propagating 
modes and the direction of propagation in the duct. Finally we can immediately carry out an approximate Wiener-Hopf factorization by inspection of (5.4). Suppose we require that

$$
\widetilde{F}(z)=\widetilde{F}_{+}(z) \widetilde{F}_{-}(z),
$$

where $\widetilde{F}_{ \pm}(z)$ is analytic and non-zero in the respective half planes $\mathfrak{I} z \gtrless 0$, then a factorization is given by

$$
\begin{aligned}
\widetilde{F}_{+}(z) & =\sqrt{F(0)} \frac{\Gamma\left(\frac{b}{a}\right)}{\Gamma\left(\frac{b+z}{a}\right)} e^{\frac{z}{2}\left[\frac{F^{\prime}(0)}{F(0)}+\psi(N+b / a) / a-\psi(P+\beta / \alpha) / \alpha\right]+\chi(z)} \\
& \times \prod_{n=1}^{N-1}\left(1+\frac{z}{z_{n}}\right) e^{-\frac{z}{z_{n}}} \\
\widetilde{F}_{-}(z) & =\sqrt{F(0)} \frac{\Gamma\left(\frac{\beta}{\alpha}\right)}{\Gamma\left(\frac{\beta-z}{\alpha}\right)} e^{\frac{z}{2}\left[\frac{F^{\prime}(0)}{F(0)}+\psi(N+b / a) / a-\psi(P+\beta / \alpha) / \alpha\right]-\chi(z)} \\
& \times \prod_{p=1}^{P-1}\left(1-\frac{z}{z_{p}}\right) e^{\frac{z}{z_{p}}}
\end{aligned}
$$

where $\chi(z)$ is an arbitrary function that can be chosen to ensure that $\widetilde{F}_{+}(z), \widetilde{F}_{-}(z)$ have suitable behaviour as $|z| \rightarrow \infty$ in appropriate half planes of analyticity.

\section{Conclusions}

We have presented a new method of $\mathrm{f}$ nite-product extraction that offers scope for useful approximations which retains the analytic properties of the original function. As an example we have presented a simple approximation for Bessel functions that is very accurate along the real axis. It is a useful and accurate approximation for f nding complex solutions of general transcendental equations involving Bessel functions. For example the method is valid mutatis-mutandis to the equation (1.1) with the derivative stroke removed. The approximation also offers simple Wiener-Hopf approximate factorizations that convey the analytic properties of the original function. Such factorizations in terms of Cauchy integral representations would be non-trivial to numerically calculate because of convergence properties of the inf nite integral. The method used here has further potential applications in that it can be applied to any special function that has an inf nite product representation whose asymptotic zeros for large argument are known. It is hoped that in the future the present approximate Wiener-Hopf method will be used on existing, and new applications, to show the accuracy and simplicity of the this method.

\section{REFERENCES}

Abramowitz, M. \& Stegun,I. A. (1964) Handbook of Mathematical Functions. New York: Dover.

Chapman, C. J. \& Sorokin, S.V. (2010) The f nite-product method in the theory of waves and stability, Proc. R. Soc., A466 (219), 471-491.

Chen, G. \& ZHOU, J. (1993) Vibration and damping in distributed systems: Vol. 2. New York: CRC Press.

Erdelyi, A.,Magnus, M,Oberhettinger,F G. \& Tricomi,F. G. (1953) Higher transcendental functions. Vol. 1 New York: McGraw-Hill. 
LAWrie, JANe B. \& ABrahams,I.DAVID. (2007) A Brief historical perspective of the Wiener-Hopf Technique, J. Eng. Math. 59, 351-358.

Rawlins, A. D. (1989) Note on the roots of $f(z)=J_{0}(z)-i J_{1}(z)$., Quart. Appl. Math., 47, 323-324.

RAWLINS, A.D. (2007) Wave propagation in a bifurcated impedance-lined cylindrical waveguide, J. Eng. Math., 59, 419-435.

Synolakis, C. M. (1988) On the roots of $f(z)=J_{0}(z)-i J_{1}(z)$., Quart. Appl. Math., 46, 105-107.

Titchmarsh,E. C. (1958) The Theory of Functions. 2nd edition. Oxford: Oxford University Press.

Watson, G. N. (1944) The Theory of Bessel Functions. Cambridge: Cambridge University Press. 